\title{
Prevalencia de depresión y factores asociados, en estudiantes de Medicina, Cuenca - Ecuador, 2019
}

\section{Prevalence of depression and associated factors, in medical students, Cuenca - Ecuador, 2019}

\author{
DOI: $10.46932 / s f j d v 3 n 2-003$
}

Received in: February $28^{\text {th }}, 2022$

Accepted in: March $1^{\text {st }}, 2022$

\section{Cinthia Gabriela Romero Sarango}

Médico General egresada de la Universidad de Cuenca - Ecuador

Dirección: Machala - El Oro - Ecuador

Correo electrónico: gabriela.romero92@ucuenca.edu.ec

Michell Susan Saavedra Palacios

Médico General egresada de la Universidad de Cuenca - Ecuador

Dirección: Cuenca - Azuay - Ecuador

Correo electrónico: michellesaavp@hotmail.com

Tania Diciana Arévalo Córdova

Médico General egresada de la Universidad Técnica de Machala - Ecuador

Dirección: Machala - El Oro - Ecuador

Correo electrónico: tarevalo_est@utmachala.edu.ec

John Jairo Molina Jaén

Médico General egresado de la Universidad Técnica de Machala - Ecuador

Universidad Técnica de Machala

Dirección: Machala - El Oro - Ecuador

Correo electrónico: john-jairo-molina@ hotmail.es

Verónica Tatiana Narea Cárdenas

Médico General egresada de la Universidad de Cuenca - Ecuador

Dirección: Cuenca - Azuay - Ecuador

Correo electrónico: veronica.nareac@ucuenca.edu.ec

\section{RESUMEN}

Introducción: La depresión contribuye significativamente a la carga mundial de morbilidad y afecta a personas de todos los países del mundo con una prevalencia global de episodios depresivos del 3,2\%. Objetivo: Determinar la prevalencia y los factores asociados a la depresión en estudiantes de la carrera de medicina de las Universidades de la ciudad de Cuenca - Azuay - Ecuador. Metodología: Estudio multicéntrico, cuantitativo, descriptivo, transversal, no experimental. La población estuvo constituida por los estudiantes de la carrera de medicina de la Universidad de Cuenca, Universidad Católica de Cuenca, Universidad del Azuay, de la ciudad de Cuenca, Provincia del Azuay - Ecuador. Para la asociación de la depresión y los factores de riesgo, se usó el Odds Ratio. Resultados: La población de estudio tuvo una media de 21 años DE \pm 2 años, con prevalencia del sexo femenino con 114 estudiantes de 272 . La prevalencia de depresión fue de $61.1 \%$. Los factores de riesgo tuvieron asociación con la depresión fueron: el sexo femenino, OR: 2,35 (IC 95\%: 1,35 - $4.86 \mathrm{p}=0,001$ ); el estar satisfecho con la carrera de medicina OR: 1,53 (IC 95\%: 1,4-1,67 p= 0,01); el recibir apoyo social OR: 1.52 (IC 95\%: 1,39- 
1,66 p= 0,03); con significancia estadística. Conclusión: Existe alta prevalencia de depresión entre los estudiantes de las Universidades de la ciudad de Cuenca - Ecuador, principalmente en el sexo femenino. Los factores que tuvieron asociación con la depresión fueron: el sexo femenino, estar satisfecho con la carrera y recibir apoyo social.

Palabras clave: depresión, factores asociados, factores de riesgo, estudiante de medicina, odds ratio.

\begin{abstract}
Introduction: Depression contributes significantly to the global burden of disease and affects people in all countries of the world with a global prevalence of depressive episodes of 3.2\%. Objective: To determine the prevalence and factors associated with depression in students of the medical career of the Universities of the city of Cuenca - Azuay - Ecuador. Methodology: Multicenter, quantitative, descriptive, crosssectional, non-experimental study. The population was constituted by students of the medical career of the University of Cuenca, Catholic University of Cuenca, University of Azuay, from the city of Cuenca, Province of Azuay - Ecuador. For the association of depression and risk factors, the Odds Ratio was used. Results: The study population had a mean of 21 years $\mathrm{SD} \pm 2$ years, with a prevalence of females with 114 students out of 272 . The prevalence of depression was $61.1 \%$. The risk factors associated with depression were: female sex, OR: $2.35(95 \% \mathrm{CI}: 1.35-4.86 \mathrm{p}=0.001)$; being satisfied with the medical degree OR: 1.53 (95\% CI: $1.4-1.67 \mathrm{p}=0.01)$; receiving social support OR: 1.52 (95\% CI: $1.39-1.66$ $\mathrm{p}=0.03$ ); with statistical significance. Conclusion: There is a high prevalence of depression among students of the Universities of the city of Cuenca - Ecuador, mainly in the female sex. The factors that were associated with depression were: being female, being satisfied with the career and receiving social support.
\end{abstract}

Keywords: depression, associated factors, risk factors, medical student, odds ratio.

\title{
1 INTRODUCCIÓN
}

La depresión, se caracteriza por el estado de abatimiento, sentimientos de tristeza, que pueden causan alteraciones de comportamiento como: disminución de la energía, pérdida de interés y del placer, sentimientos de culpa o baja estima, alteración del sueño, apetito y falta de concentración (Vargas et al, 2017; Rodríguez-Castro, et al. 2018).

La prevalencia mundial del trastorno depresivo varía del 8 al 25\% en la población, con una relación mujer - hombre de 2:1, con promedio de edad entre 20 y 45 años (Organización Mundial de la Salud OMS, 2001); contribuyendo significativamente a la carga mundial de morbilidad, con una prevalencia global de episodios depresivos del 3,2\%. Estos trastornos depresivos generalmente comienzan a una edad temprana y son recurrentes a lo largo de la vida (Cui, 2016; Ngasa et al. 2017).

Según el Ministerio de Salud Pública MSP (2022), en el 2015, se registraron 50.379 personas con diagnóstico presuntivo y definitivo por depresión; de las cuales, 36.631 corresponden a mujeres y 13.748 a hombres, con 3406 hospitalizaciones relacionadas con casos de depresión.

En un estudio realizado en estudiantes foráneos de la Universidad Católica de Cuenca, en el 2019, la prevalencia de depresión fue de $62.6 \%$, siendo los factores asociados a la depresión: el sexo femenino 
(OR: 2,60 IC 95\%: 1,39 - 4,86 p= 0,002); tener poco o ningún apoyo social (OR: 9,56 IC 95\%: 0,54 $-170,06 \mathrm{p}=0,04)$; y no tener pareja civil (OR: 4,25 IC 95\%: 1,06 - 17,01 p=0,03), todos con significancia estadística (Romero, et al. 2022).

Se considera que la prevalencia de los trastornos mentales es más frecuente en poblaciones con características particulares, entre las que se encuentran los estudiantes de la carrera de medicina, debido a las altas exigencias sociales, psicológicas y académicas que demanda esta carrera (Roman et al, 2008, Dyrbye, et al. 2005).

Dentro de los factores a los que se encuentran expuestos está la excesiva carga de trabajo, las pérdidas académicas, e la falta de apoyo económico, problemas de adaptación a otra ciudad, el estado civil, entre otros (Roman et al, 2008, Dyrbye, et al. 2005; Benbassat et al, 2011; Gonzalez-Olaya et al. 2014, Romero, et al. 2022) .

Por estos antecedentes, el objetivo del presente estudio es determinar la prevalencia y los factores asociados a la depresión en estudiantes de la carrera de medicina de las Universidades de la ciudad de Cuenca - Azuay - Ecuador.

\section{MATERIALES Y MÉTODOS}

Se realizó un estudio multicéntrico, cuantitativo, descriptivo, transversal, no experimental. La población estuvo constituida por los estudiantes de la carrera de medicina de la Universidad de Cuenca, Universidad Católica de Cuenca, Universidad del Azuay, de la ciudad de Cuenca, Provincia del Azuay Ecuador, de primero a décimo semestre del periodo lectivo Marzo - Julio de 2019.

Se realizó la evaluación del apoyo social mediante la escala de apoyo social (AFA-R15) y la evaluación de la depresión se realizó mediante el test de Hamilton. Para la asociación de la depresión y los factores asociados, los datos fueron dicotomizados y como medida de asociación se usó el Odds Ratio, calculando la significancia estadística mediante el $\mathrm{Chi}^{2}$.

Para la realización del estudio, se obtuvo el permiso de los Decanos de la Facultad de Medicina de las Universidades mencionadas y el consentimiento informado de los informados, previo a la aplicación del instrumento de recolección de datos y aplicación de la escala de apoyo social y test de Hamilton. Para el análisis estadístico se usó el software IBM Statistical Package for the Social Sciences (SPSS) versión 26. 


\section{RESULTADOS}

La población estuvo constituida por 272 estudiantes de medicina, en un rango de 18 a 30 años, con una media de 22 años $\mathrm{DE} \pm 2$ años, siendo la edades con mayor prevalencia, 21 y 22 años, con 61 y 55 estudiantes, respectivamente (Figura 1).

Figura 1 Edad de estudiantes de medicina de las Universidades de la ciudad de Cuenca

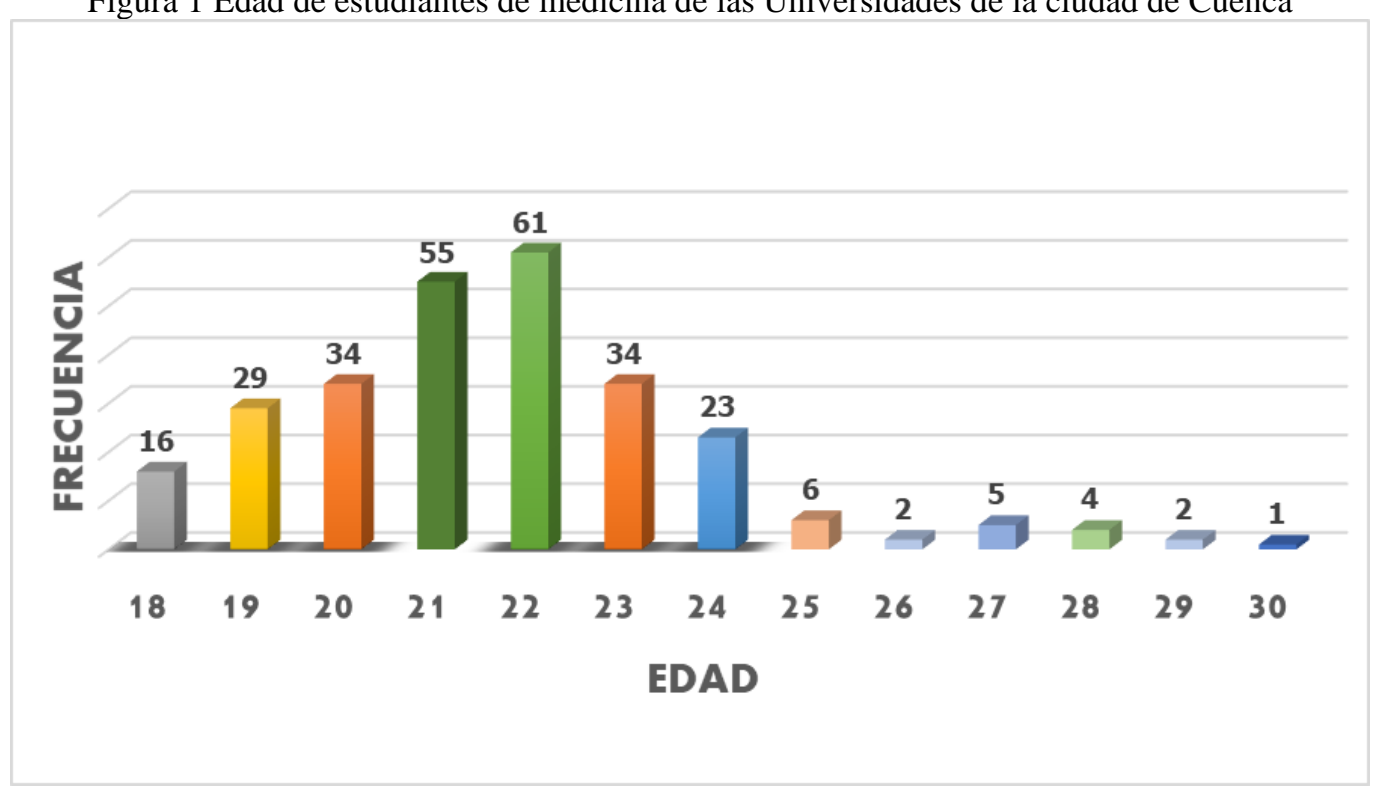

El sexo con mayor prevalencia fue el femenino con 178 estudiantes, mientras que 94 estudiantes eran del sexo masculino; el rango de edad prevalente fue de 18 a 21 años con un $49.3 \%$; siendo la mayoría de estudiantes de estado civil soltero, con un 92,3\%; el semestre con mayor número de estudiantes fue de 7 a 8vo semestre; 182 estudiantes pertenecían a la Universidad Católica de Cuenca (66,9\%), 76 pertenecían a la Universidad de Cuenca (27.9\%) y 14 a la Universidad del Azuay (5,1\%); el 87,5\% de estudiantes eran financiados sus estudios por sus padres ( (Tabla 1). 
Tabla 1. Datos sociodemográficos de los estudiantes de medicina de las Universidades de Cuenca

\begin{tabular}{|c|c|c|c|c|c|c|}
\hline \multirow[t]{2}{*}{ Variable } & \multicolumn{2}{|c|}{$\frac{\text { Masculino }}{(n=68)}$} & \multicolumn{2}{|c|}{$\frac{\text { Femenino }}{(n=114)}$} & \multicolumn{2}{|c|}{$\underline{\text { Total }}(\mathbf{n = 2 7 2 )}$} \\
\hline & $\mathbf{f}$ & $\%$ & $\mathbf{f}$ & $\%$ & $\bar{f}$ & $\%$ \\
\hline \multicolumn{7}{|l|}{ Rango de edad } \\
\hline $18-21$ & 42 & $61,8 \%$ & 92 & $80,7 \%$ & 134 & $49,3 \%$ \\
\hline $22-25$ & 45 & $66,2 \%$ & 79 & $69,3 \%$ & 124 & $45,6 \%$ \\
\hline $26-29$ & 6 & $8,8 \%$ & 7 & $6,1 \%$ & 13 & $4,8 \%$ \\
\hline$\geq 30$ & 1 & $1,5 \%$ & 0 & $0,0 \%$ & 1 & $0,4 \%$ \\
\hline \multicolumn{7}{|l|}{ Estado Civil } \\
\hline casado/a & 6 & $8,8 \%$ & 9 & $7,9 \%$ & 15 & $5,5 \%$ \\
\hline unión libre & 1 & $1,5 \%$ & 4 & $3,5 \%$ & 5 & $1,8 \%$ \\
\hline soltero/a & 86 & $126,5 \%$ & 165 & $144,7 \%$ & 251 & $92,3 \%$ \\
\hline divorciado/a & 1 & $1,5 \%$ & 0 & $0,0 \%$ & 1 & $0,4 \%$ \\
\hline Semestre que cursa & & $0,0 \%$ & & & & \\
\hline 1-2 semestre & 16 & $23,5 \%$ & 26 & $22,8 \%$ & 42 & $15,4 \%$ \\
\hline 3-4 semestre & 26 & $38,2 \%$ & 47 & $41,2 \%$ & 73 & $26,8 \%$ \\
\hline 5-6 semestre & 17 & $25,0 \%$ & 26 & $22,8 \%$ & 43 & $15,8 \%$ \\
\hline $7-8$ semestre & 25 & $36,8 \%$ & 54 & $47,4 \%$ & 79 & $29,0 \%$ \\
\hline $9-10$ semestre & 10 & $14,7 \%$ & 25 & $21,9 \%$ & 35 & $12,9 \%$ \\
\hline \multicolumn{7}{|l|}{ Universidad a la que pertenece } \\
\hline Universidad Católica de Cuenca & 68 & $100,0 \%$ & 114 & $100,0 \%$ & 182 & $66,9 \%$ \\
\hline Universidad del Azuay & 3 & $4,4 \%$ & 11 & $9,6 \%$ & 14 & $5,1 \%$ \\
\hline Universidad de Cuenca & 23 & $33,8 \%$ & 53 & $46,5 \%$ & 76 & $27,9 \%$ \\
\hline \multicolumn{7}{|l|}{ Financiamiento } \\
\hline Padres & 78 & $114,7 \%$ & 160 & $140,4 \%$ & 238 & $87,5 \%$ \\
\hline Auto financiamiento & 8 & $11,8 \%$ & 4 & $3,5 \%$ & 12 & $4,4 \%$ \\
\hline Beca o crédito estudiantil & 5 & $7,4 \%$ & 9 & $7,9 \%$ & 14 & $5,1 \%$ \\
\hline Otros & 3 & $4,4 \%$ & 5 & $4,4 \%$ & 8 & $2,9 \%$ \\
\hline
\end{tabular}

La prevalencia de depresión en estudiantes, fue 182 estudiantes (62.6\%), presentando mayor porcentaje de depresión los estudiantes de la Universidad del Azuay, 11 estudiantes con depresión de 14 estudiantes (78,6\%), seguido por los estudiantes de la Universidad de Cuenca, con 57 estudiantes con depresión de 76 estudiantes (75\%). En relación a la evaluación de la depresión, el 26,1\% presentaron depresión leve, el 18\% moderada (Tabla 2).

Tabla 2 Prevalencia de Depresión en estudiantes de las Universidades de Cuenca

\begin{tabular}{|c|c|c|c|c|c|c|c|c|}
\hline \multirow[t]{2}{*}{ Variable } & \multicolumn{2}{|c|}{$\begin{array}{c}\begin{array}{c}\text { Universidad } \\
\text { Católica de Cuenca }\end{array} \\
(n=182)\end{array}$} & \multicolumn{2}{|c|}{$\begin{array}{l}\text { Universidad de } \\
\text { Cuenca } \\
(n=76) \\
\end{array}$} & \multicolumn{2}{|c|}{$\begin{array}{l}\text { Universidad del } \\
\qquad \begin{array}{c}\text { Azuay } \\
(\mathrm{n}=14)\end{array}\end{array}$} & \multicolumn{2}{|c|}{ Total } \\
\hline & $\mathbf{f}$ & $\%$ & f & $\%$ & $\mathbf{f}$ & $\%$ & $\mathbf{f}$ & $\%$ \\
\hline \multicolumn{9}{|l|}{ Depresión } \\
\hline $\mathrm{Si}$ & 114 & $62,6 \%$ & 57 & $75,0 \%$ & 11 & $78,6 \%$ & 182 & $66,9 \%$ \\
\hline No & 35 & $19,2 \%$ & 19 & $25,0 \%$ & 3 & $21,4 \%$ & 57 & $21,0 \%$ \\
\hline \multicolumn{9}{|l|}{ Evaluación de depresión } \\
\hline Sin depresión & 68 & $37,4 \%$ & 19 & $25,0 \%$ & 3 & $21,4 \%$ & 90 & $33,1 \%$ \\
\hline Depresión leve & 50 & $27,5 \%$ & 13 & $17,1 \%$ & 8 & $57,1 \%$ & 71 & $26,1 \%$ \\
\hline Depresión moderada & 30 & $16,5 \%$ & 18 & $23,7 \%$ & 1 & $7,1 \%$ & 49 & $18,0 \%$ \\
\hline Depresión grave & 17 & $9,3 \%$ & 14 & $18,4 \%$ & 1 & $7,1 \%$ & 32 & $11,8 \%$ \\
\hline Depresión severa & 17 & $9,3 \%$ & 12 & $15,8 \%$ & 1 & $7,1 \%$ & 30 & $11,0 \%$ \\
\hline
\end{tabular}

En relación a los factores asociados, que se presentan con mayor frecuencia en los estudiantes que presentan depresión, tenemos: financiamiento de los estudios por parte de terceros, 95,6\%; recibir 
apoyo social, 95,10\%; no tener pareja civil, 94\%; sentirse muy satisfecho con la carrera de medicina, 93,4\%; no consumir drogas, 87,9\% y tener una edad entre 18 a 23 años, 85.2\% (Tabla 3).

Tabla 3 Factores asociados a la depresión en estudiantes con depresión de las Universidades de Cuenca

\begin{tabular}{|c|c|c|c|c|c|c|c|c|}
\hline \multirow[t]{2}{*}{ Factor Asociado } & \multicolumn{2}{|c|}{$\begin{array}{c}\text { Universidad } \\
\text { Católica de Cuenca } \\
(n=114)\end{array}$} & \multicolumn{2}{|c|}{$\begin{array}{l}\text { Universidad de } \\
\text { Cuenca } \\
(n=57)\end{array}$} & \multicolumn{2}{|c|}{$\begin{array}{l}\text { Universidad del } \\
\qquad \frac{\text { Azuay }}{(\mathrm{n}=11)}\end{array}$} & \multicolumn{2}{|c|}{ Total } \\
\hline & $\mathbf{f}$ & $\%$ & f & $\%$ & $\mathbf{f}$ & $\%$ & $\mathbf{f}$ & $\%$ \\
\hline \multicolumn{9}{|l|}{ Sexo } \\
\hline Femenino & 81 & $71,1 \%$ & 41 & $71,9 \%$ & 9 & $81,8 \%$ & 131 & $72,0 \%$ \\
\hline Masculino & 33 & $28,9 \%$ & 16 & $28,1 \%$ & 2 & $18,2 \%$ & 51 & $28,0 \%$ \\
\hline \multicolumn{9}{|l|}{ Edad } \\
\hline 18 a 23 años & 100 & $87,7 \%$ & 46 & $80,7 \%$ & 9 & $81,8 \%$ & 155 & $85,2 \%$ \\
\hline$\geq 24$ años & 14 & $12,3 \%$ & 11 & $19,3 \%$ & 2 & $18,2 \%$ & 27 & $14,8 \%$ \\
\hline \multicolumn{9}{|l|}{ Estado Civil } \\
\hline Sin Pareja civil & 111 & $97,4 \%$ & 49 & $86,0 \%$ & 11 & $100,0 \%$ & 171 & $94,0 \%$ \\
\hline Con Pareja civil & 3 & $2,6 \%$ & 8 & $14,0 \%$ & 0 & $0,0 \%$ & 11 & $6,0 \%$ \\
\hline \multicolumn{9}{|l|}{ Tipo de familia } \\
\hline Monoparental & 38 & $33,3 \%$ & 21 & $36,8 \%$ & 2 & $18,2 \%$ & 61 & $33,5 \%$ \\
\hline Biparental & 76 & $66,7 \%$ & 36 & $63,2 \%$ & 9 & $81,8 \%$ & 121 & $66,5 \%$ \\
\hline \multicolumn{9}{|l|}{ Con quien vive } \\
\hline Solo & 37 & $32,5 \%$ & 30 & $52,6 \%$ & 2 & $18,2 \%$ & 69 & $37,9 \%$ \\
\hline Acompañado & 77 & $67,5 \%$ & 27 & $47,4 \%$ & 9 & $81,8 \%$ & 113 & $62,1 \%$ \\
\hline \multicolumn{9}{|l|}{ Año que cursa } \\
\hline 3 primeros años & 66 & $57,9 \%$ & 40 & $70,2 \%$ & 6 & $54,5 \%$ & 112 & $61,5 \%$ \\
\hline 2 últimos años & 48 & $42,1 \%$ & 17 & $29,8 \%$ & 5 & $45,5 \%$ & 70 & $38,5 \%$ \\
\hline \multicolumn{9}{|l|}{ Satisfacción con la carrera } \\
\hline Poco o nada satisfecho & 6 & $5,3 \%$ & 6 & $10,5 \%$ & 0 & $0,0 \%$ & 12 & $6,6 \%$ \\
\hline $\begin{array}{l}\text { Moderada - muy } \\
\text { satisfecho }\end{array}$ & 108 & $94,7 \%$ & 51 & $89,5 \%$ & 11 & $100,0 \%$ & 170 & $93,4 \%$ \\
\hline \multicolumn{9}{|l|}{ Carga horaria } \\
\hline$>8$ horas & 37 & $32,5 \%$ & 30 & $52,6 \%$ & 10 & $90,9 \%$ & 77 & $42,3 \%$ \\
\hline$<8$ horas & 77 & $67,5 \%$ & 27 & $47,4 \%$ & 1 & $9,1 \%$ & 105 & $57,7 \%$ \\
\hline \multicolumn{9}{|l|}{ Apoyo social } \\
\hline Poco o ninguno & 7 & $6,1 \%$ & 2 & $3,5 \%$ & 0 & $0,0 \%$ & 9 & $4,9 \%$ \\
\hline Si recibe & 107 & $93,9 \%$ & 55 & $96,5 \%$ & 11 & $100,0 \%$ & 173 & $95,1 \%$ \\
\hline \multicolumn{9}{|l|}{ Financiamiento } \\
\hline Autofinanciado & 5 & $4,4 \%$ & 3 & $5,3 \%$ & 0 & $0,0 \%$ & 8 & $4,4 \%$ \\
\hline Financiado por terceros & 109 & $95,6 \%$ & 54 & $94,7 \%$ & 11 & $100,0 \%$ & 174 & $95,6 \%$ \\
\hline \multicolumn{9}{|l|}{ Consumo de drogas } \\
\hline $\mathrm{Si}$ & 10 & $8,8 \%$ & 12 & $21,1 \%$ & 0 & $0,0 \%$ & 22 & $12,1 \%$ \\
\hline No & 104 & $91,2 \%$ & 45 & $78,9 \%$ & 11 & $100,0 \%$ & 160 & $87,9 \%$ \\
\hline
\end{tabular}

$\mathrm{n}=182$

Los factores que tuvieron asociación con la depresión, sin significancia estadística fueron: el sexo femenino, OR: 2,35 (IC 95\%: 1,35-4.86 $p=0,001$ ); el estar satisfecho con la carrera de medicina OR: 1,53 (IC 95\%: 1,4 - 1,67 p=0,01); el recibir apoyo social OR: 1.52 (IC 95\%: 1,39 - 1,66 p=0,03); con significancia estadística. Mientras que el tener una carga horaria menor a 8 horas se mostró como un efecto protector OR: 0.876 (IC 95\%: 0,53 - 1,46 p=0,61), pero sin significancia estadística. 
Tabla 4 Asociación de factores con la depresión en estudiantes de las Universidades de Cuenca

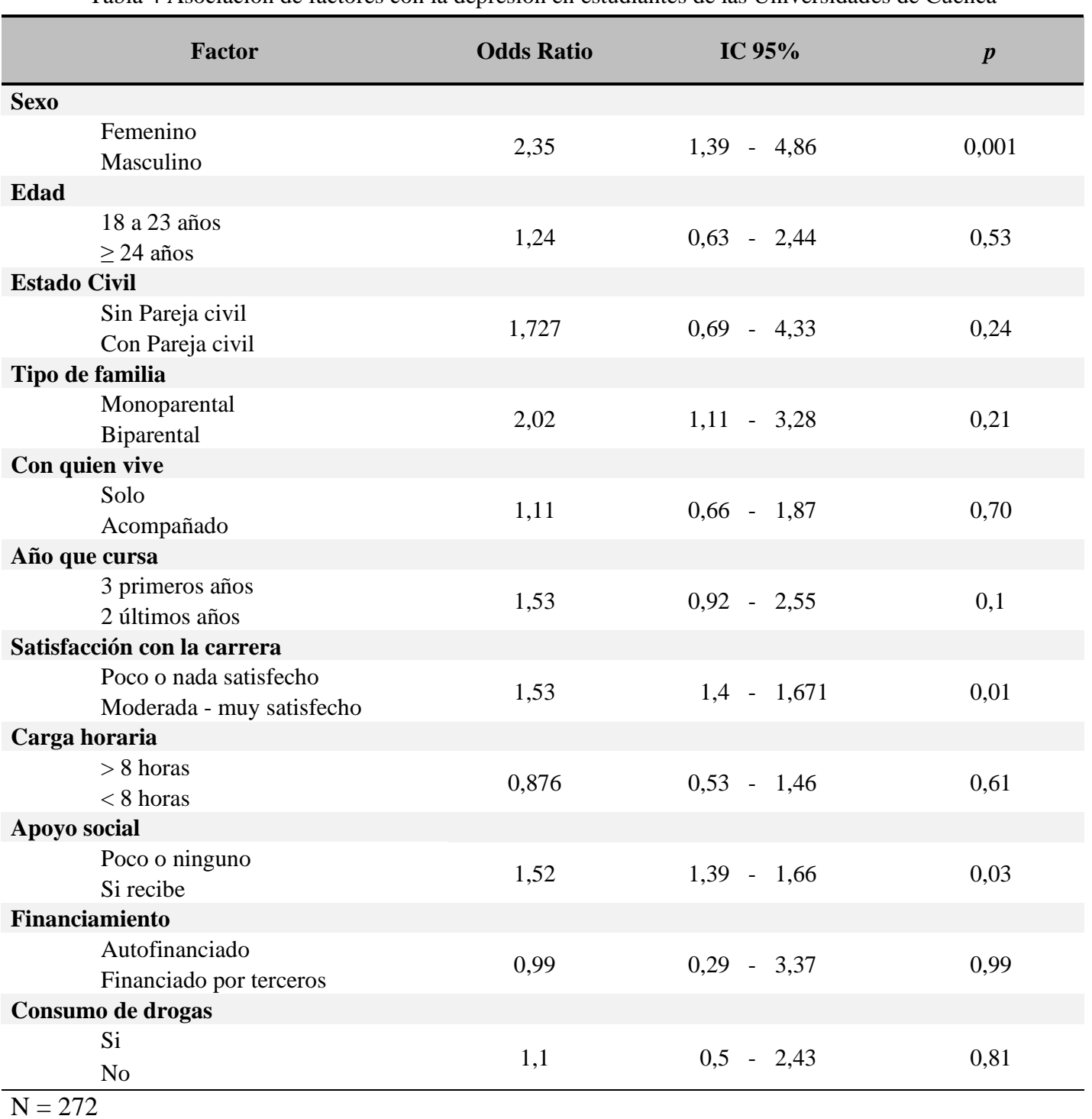

\section{DISCUSIÓN}

En el presente estudio, la prevalencia en los estudiantes fue muy elevada, con un $62.6 \%$, en contraposición con los estudio realizados por Bernita et al (2017), en el cual los estudiantes de medicina de la Universidad Católica de Cuenca - Ecuador, presentó el $47.7 \%$ de depresión; en el Estudio realizado por Chapilliquen R. realizado en Perú, en estudiantes Universitarios foráneos de la Universidad Privada Antenor Orrego, Trujillo- Perú, en donde la prevalencia fue de 22.91\%; y, el estudio realizado por Martínez M., en estudiantes universitarios de la carrea de Medicina, de la Universidad de Puebla México, donde la depresión fue de $31.72 \%$.

El sexo femenino fue el de mayor prevalencia en depresión con un $71,1 \%$, teniendo el sexo femenino asociación con la depresión, con un OR: 2,60 (IC 95\%: 1,39-4,86 p= 0,002), en concordancia con los estudios realizados por Rodríguez (2018), en el cual el 57.55\% tenían depresión; Obregon et al. (2020), en donde el sexo femenino presentó un factor de riesgo de 1,03 veces más para padecer depresión; 
y, el estudio de Romero et al (2022), en estudiantes de medicina de la Universidad Católica de Cuenca, en el cual el 62,2\% de las mujeres presentaron depresión, con un (OR: 2,60 IC 95\%: 1,39-4,86 p= $0,002)$.

\section{CONCLUSIÓN}

Existe alta prevalencia de depresión entre los estudiantes de las Universidades de la ciudad de Cuenca - Ecuador, principalmente en el sexo femenino y en los estudiantes de 18 a 24 años. Los factores que tuvieron asociación con la depresión fueron: el sexo femenino, estar satisfecho con la carrera y recibir apoyo social, con significancia estadística. Los factores protectores encontrados fueron tener una carga horaria menor a 8 horas, pero sin significancia estadística. 


\section{REFERENCIAS}

Bernita E., Castillo F. y Vallejo E. (27 de julio de 2017) Prevalencia de depresión y factores asociados en los estudiantes de Medicina de la Universidad Católica de Cuenca. 2014. Rev Fac Cienc Médicas Univ Cuenca; 35(1):68-73.

Disponible

en: https://publicaciones.ucuenca.edu.ec/ojs/index.php/medicina/article/view/1240/1078

Benbassat J, Baumal R, Chan S, Nirel N. (2005) Sources of distress during medical training and clinical practice: suggestions for reducing their impact. Med Teach, 33: 486-90.

Cui R. (2015) Editorial (Selección temática: una revisión sistemática de la depresión), Neurofarmacología actual, 13(4). Disponible en: https://dx.doi.org/10.2174/1570159X1304150831123535

Dyrbye LN, Thomas MR, Shanafelt TD. Medical student distress: causes, consequences, and proposed solutions. Mayo Clin Proc. 2005 Dec;80(12):1613-22. Available in: https://dx.doi.org/10.4065/80.12.1613

González-Olaya, H., Delgado-Rico, H., Escobar-Sánchez, M. y Cárdenas- Angelone, M. (2014). Asociación entre el estrés, el riesgo de depresión y el rendimiento académico en estudiantes de los primeros semestres de un programa colombiano de medicina. FEM: Revista de la Fundación Educación Médica, 17(1), 47-54. Disponible en: https://dx.doi.org/10.4321/S2014-98322014000100008

Jacobs G. (2002) Non academic factors affecting the academic success of Grenadian students at St. George's University. St. George's: University Centre; 2002

Martínez-Martínez M del C, Muñoz-Zurita G, Rojas-Valderrama K, Sánchez-Hernández JA. (2016) Prevalencia de síntomas depresivos en estudiantes de la licenciatura en Medicina de Puebla, México. Aten Fam, 23(4),145- $149 . \quad$ Disponible en: https://www.sciencedirect.com/science/article/pii/S140588711630150X

Ministerio de Salud Pública (26 de enero de 2022). Este 7 de abril se celebra el Día Mundial de la Salud, con el tema "Depresión: Hablemos". Noticias Destacadas. Disponible en: https://www.salud.gob.ec/este7-de-abril-se-celebra-el-dia-mundial-de-la-salud-con-el-tema-depresion-hablemos/

Ngasa, SN, Sama, CB., Dzekem, BS et al. (2017). Prevalencia y factores asociados con la depresión entre estudiantes de medicina en Camerún: un estudio transversal. BMC Psiquiatría 17, 216. Disponible en: https://doi.org/10.1186/s12888-017-1382-3

Obregón-Morales, Berea, Montalván-Romero, José C., Segama-Fabian, Edinho, Dámaso-Mata, Bernardo, Panduro-Correa, Vicky, \& Arteaga-Livias, Kovy. (2020). Factors associated with depression in medical students from a peruvian university. Educación Médica Superior, 34(2), e1881. Epub 01 de junio de 2020. Recuperado en 27 de enero de 2022, de http://scielo.sld.cu/scielo.php?script=sci_arttext\&pid=S0864-21412020000200013\&lng=es\&tlng=en.

Organización Mundial de la Salud OMS. (13 de Septiembre de 2021) Depresión. Centro de prensa. Disponible en: https://www.who.int/es/news-room/fact-sheets/detail/depression

Organización Mundial de la Salud. (2001) Informe sobre la salud en el mundo 2001: Salud mental : nuevos conocimientos, nuevas esperanzas. Organización Mundial de la Salud. Disponible en: https://apps.who.int/iris/handle/10665/42447 
Ríos Chapilliquen, E. J. (2018) Antecedente de migración como factor asociado a depresión en estudiantes del primer año de medicina. Disponible en: http://repositorio.upao.edu.pe/handle/20.500.12759/4008

Rodríguez-Castro AI, Ríos-González CM.(2018) Frecuencia de depresión en estudiantes de medicina.Medicina Clínica y Social, 2018;2(3):128-135. Disponible en: https://www.medicinaclinicaysocial.org/index.php/MCS/article/view/60/57

Romero C., et al. (2022) Factores asociados a la depresión en estudiantes foráneos de Medicina, Universidad Católica de Cuenca, 2019. South Florida Journal of Development, Miami,3 (1), 906-913. Disponible en: https://doi.org/10.46932/sfjdv3n1-069

Vargas M, et al. (2017) Influencia de los hábitos en la depresión del estudiante de medicina peruano: estudio en siete departamentos. Rev Colomb Psiquiat. http://dx.doi.org/10.1016/j.rcp.2017.01.008

en: 